\title{
EXCITABLE PULSES AND DIFFUSION OF LOCALIZED STATES IN A DRIVEN SEMICONDUCTOR LASER WITH DELAY
}

\author{
Bruno Garbin \\ The Dodd-Walls Centre for Photonic and \\ Quantum Technologies, Department of Physics \\ The University of Auckland \\ Auckland 1142, New Zealand \\ b.garbin@auckland.ac.nz
}

\author{
Giovanna Tissoni \\ Université Côte d'Azur - CNRS \\ Institut de Physique de Nice \\ France \\ giovanna.tissoni@unice.fr
}

\author{
Stephane Barland \\ Université Côte d'Azur - CNRS \\ Institut de Physique de Nice \\ France \\ stephane.barland@unice.fr
}

Article history:

Received 15.05.2018, Accepted 15.09.2018

\begin{abstract}
Semiconductor lasers with optical injection may be brought to an "excitable" regime, in which they respond to external perturbations in a neuron-like way. When submitted to delayed optical feedback this system can host stable optical localized states. We characterize experimentally the excitable response of a semiconductor laser with optical injection to external perturbations for different parameter values and show that localized states may diffuse in presence of noise.
\end{abstract}

\section{Key words}

Delay, excitability, noise, localized states, random walk, laser.

\section{Introduction}

As an important biophysical process, allowing the heart to beat or the transport of the information in the brain, excitability has been intensively studied during the past fifty years. It has been found in biology [Hodgkin et al., 1952; Hodgkin and Huxley, 1952], in chemistry [Neumann and Bernhardt, 1977; Kuhnert L. et al., 1989], and in optical systems like in laser with saturable absorber [Plaza et al., 1997; Dubbeldam et al., 1999; Larotonda et al., 2002], lasers with optical feedback [Giudici et al., 1997] and lasers with optical injection [Wieczorek et al., 2002; Goulding et al., 2007; Kelleher et al., 2011;
Barland et al., 2003; Turconi et al., 2013].

Excitability is defined as an all or nothing response under an external perturbation. If the perturbation is large enough to overcome the excitability threshold, the system responds in a unique and well defined way due to a strongly attractive trajectory in the phase space. Otherwise the system only relaxes, following a response linearly dependent on the perturbation. A Laser with injected signal can be brought into an excitable regime, when injectionlocked to the forcing and in the vicinity of a saddlenode on a circle bifurcation (as unlocking transition) [Coullet et al., 1998]. The threshold separating the two responses can be observed by applying different kinds of perturbation such as applying a short bias current pulse [Turconi et al., 2013], injecting an incoherent optical perturbation [Garbin et al., 2014], or perturbing the phase of the injected field [Turconi et al., 2013; Garbin et al., 2013]. This last method has been found to be the most efficient, which can be understood considering that this system is essentially a phase oscillator well described by the Adler equation [Adler, 1946].

Excitability in optics has been also intensively studied due to the large number of possible applications, among which we can cite the possibility to build neural computing architectures [Maass and Markram, 2004], or to realize wavelength conversion and pulse reshaping [Garbin et al., 2014] due to the unicity of the response, which does not depend on the perturbation. Recently the storage and individual addressing of optical phase bits has been demonstrated in an experimental system based on laser with injected signal in 
the excitable regime [Garbin et al., 2015]. In that experiment, excitable orbits determined by the topology of the phase space are foundational for the stability of the dissipative localized states which actually carry the information. In all these cases, the characterization of the properties of the excitable pulses and their dependence on experimental parameters is a required step.

In the following we analyze experimentally the excitable response of a semiconductor laser to perturbations in the injection beam phase and show that responses as short as $10 \mathrm{ps}$ can be observed, but that intensity dynamics (in addition to phase dynamics) becomes increasingly relevant for faster and faster responses.

\section{Experiment}

The experimental setup is essentially the same as described in [Turconi et al., 2013]. It consists of a laser with an injected signal experiment based on a VCSEL (ULM980-03-TN-S46, the Slave laser) purely single(transverse and longitudinal) mode and linearly polarized (up to $1.8 \mathrm{~mA}$ ). It emits at $980 \mathrm{~nm}$ and its coherent emission threshold $\left(I_{t h}\right)$ is around $0.2 \mathrm{~mA}$. In the following the Slave laser will be electrically pumped at about $7-8 \times I_{t h}$ to keep the system in the simplest mode of operation, ie purely single transverse mode, linearly polarized and strongly damped relaxation oscillations.

The injection laser (the Master laser) is an edgeemitting laser whose emission wavelength is step-wise tunable thanks to optical feedback via an external grating. It is isolated from the Slave laser by a 40-dB optical isolator to avoid bidirectional coupling. In order to apply perturbations we place on the injection path a (polarization preserving) $10 \mathrm{GHz}$ fiber coupled Lithium Niobate phase modulator (to apply phase perturbations as investigated in [Turconi et al., 2013]). The fibered output of the modulator is connected to a fibered 10/90 beam splitter, the lower power beam being used for high-bandwidth monitoring of the master beam intensity. The high-power beam is then directed to a half-wave plate and polarizing beam splitter which enable setting the portion of the master beam which will finally reach the slave laser through a $10 \%$ reflective beam-splitter.

The two lasers are temperature stabilized within $0.01^{\circ} \mathrm{K}$ by Peltier elements. The Slave laser is protected from unwanted backreflections on the measurement apparatus by an optical isolator. The output beam is then split using a half-wave plate and a polarizing beam-splitter. One part is directed to a Fabry-Perot interferometer (72-GHz free spectral range, Finesse 110), which allows monitoring the frequency detuning $\Delta$, defined as the frequency of Master laser minus that of the Slave. The other part is coupled into an optical fiber which guides light to an ultrafast photodetector (Thorlabs 9-GHz bandwidth or Newfocus $35 \mathrm{GHz}$ band-

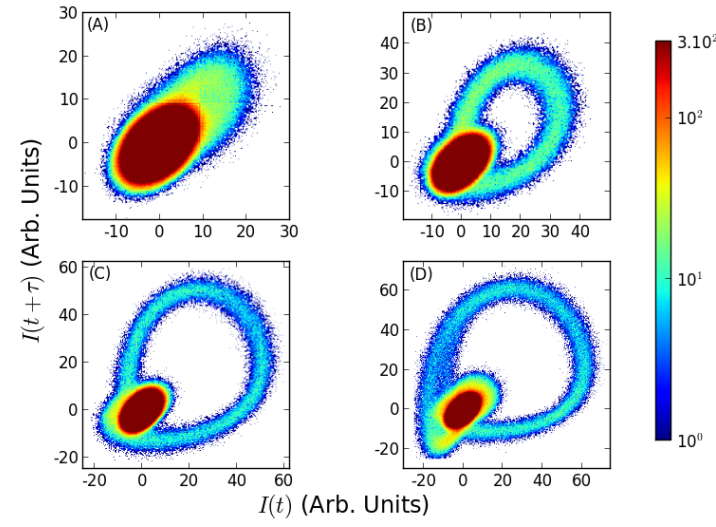

Figure 1. Bidimensional projection of the phase space trajectory of the system under perturbations in the phase of the master beam for different parameters. The phase space reconstruction is obtained by time-delay embedding with a delay of $30 \mathrm{ps}$ and is plotted for about 5000 realizations. The color scale is logarithmic. (A): $I_{s l}=$ $1.336 \mathrm{~mA}, P_{i n j}=1 \mu \mathrm{W}, \Delta=-3.8 \mathrm{GHz}$; (B): $I_{s l}=$ $1.320 \mathrm{~mA}, P_{i n j}=3 \mu \mathrm{W}, \Delta=-6.3 \mathrm{GHz}$; (C): $I_{s l}=$ $1.299 \mathrm{~mA}, P_{i n j}=7 \mu \mathrm{W}, \Delta=-8.9 \mathrm{GHz}$; (D): $I_{s l}=$ $1.295 \mathrm{~mA}, P_{i n j}=8.5 \mu \mathrm{W}, \Delta=-10 \mathrm{GHz}$. Acquired with detection Path 1.

width). After further amplification (via $12 \mathrm{GHz}$ or 40 $\mathrm{GHz}$ bandwidth RF amplifiers), the signal is acquired by a real-time oscilloscope (respectively: DPO71254C $12.5 \mathrm{GHz}, 100 \mathrm{GS} / \mathrm{s}$ (Path 1) or DPO73304D $33 \mathrm{GHz}$, $100 \mathrm{GS} / \mathrm{s}$ (Path 2)). The first detection path (detection Path 1 in the following) allows better detection of small pulses due to higher sensitivity, at the cost of less bandwidth with respect to detection Path 2.

\subsection{Characterization of Excitable Spikes}

In order to analyze the properties of the excitable response in different parameter regimes (essentially injected power and detuning), the response of the system to phase perturbations of the injected field is observed in a time-delay phase space reconstruction of the phase space trajectory. This perturbation, (rising and falling steps of $100 \mathrm{ps}$ in the phase of the Master beam) whose mechanism is described in [Turconi et al., 2013], may reach 100\% efficiency for the highest amplitudes [Garbin et al., 2013]. On Figure1, we show a bidimensional projection of the phase space obtained via time-delay reconstruction method with a delay of $30 \mathrm{ps.} \mathrm{With} \mathrm{this} \mathrm{method} \mathrm{(plotting}$ the time series as a function of a time-shifted copy of itself), the phase space structure can be reconstructed from a univariate time series analysis (see [Takens, 1981; Kennel et al., 1992]. The logarithmic color code indicates how often a particular region of the phase space is visited. Although the logarithmic color coding is a not very easy to read, it is extremely useful to show how attractive the excitable orbit is. It is 
a key property of excitable systems to exhibit very well defined responses to perturbations and here the very sharply defined reconstructed phase space trajectory neatly demonstrates its strongly attractive nature. Although this phase space reconstruction approach does not preserve all the geometrical properties of the phase space, applying always the same embedding procedure neatly shows the evolution of the excitable orbit with parameters. For that measurement we used the detection Path 1. The four situations described here correspond to four diffent detunings $(-3.8,-6.3,-8.9$ and $-10 \mathrm{GHz}$ ). In each case, the injected power $P_{i n j}$ was set such that the system is as close as possible to the unlocking transition. A stable fixed point is visible in each panel of Figure1 as a deep red point, very often visited by the system.

The system is more sensitive with the rising front of the perturbation due to the negative detuning used and to the asymmetrical positions of the fixed points on the Adler circle [Adler, 1946]. On Figure1(A), only a relatively small bump at the top right of the fixed point is visible, indicating that the response of the system takes place in the phase of the field at almost constant intensity [Kelleher et al., 2011]. With Figure1(B)-(D) we remark the appearance of a large and unique trajectory outside of the stable fixed point with a little dispersion due to the detection noise: the excitable response.

Figure1(D) is plotted for a larger perturbation as compared to the other images. It is then possible to observe a small ring at the top right of the fixed point corresponding to the linear response of the system to the inverted perturbation (falling front). From these measurements, one concludes that larger injected power (with adjusted detuning so as to keep the system as close as possible to the unlocking transition) leads to a larger and larger intensity signature of the excitable orbit.

Moreover Figure1(C) and Figure1(D) exhibit at the bottom left of the fixed point a little broadening corresponding to the relaxation of the system towards the fixed point after an excitable excursion. One remarks the increase of this part of the response in the same way as the general intensity signature of the excitable orbit. Furthermore, the broadening in the lower left corner may be related to relaxation oscillations, excited in response to the strong variation of field intensity. An attentive examination of panels (B)-(D) of Figure1 reveals a weaker and weaker density along the excitable orbit, which suggests less visited regions of the phase space. Since all panels were plotted for essentially identical number of realizations (close to 5000) and unity efficiency, one concludes that this visual effect is a result of faster trajectories. Indeed, due to the finite sampling rate of the oscilloscope (100 GS/s), it results in a smaller number of sampling points within one orbit.

This can be further studied by measuring the duration of the excitable orbit depending on parameters. Due to the nature of the excitable orbit (which corresponds to a temporary unlocking of the Slave laser's phase) one expects this duration to scale roughly linearly with the inverse of the detuning between Slave and Master lasers. Accordingly, we show on Figure 2 the evolution of excitable pulses duration with the detuning $\Delta$. This latter has been built in the same way as Figure1, setting simultaneously $P_{i n j}$ and $\Delta$ to keep the system as close as possible to the unlocking boundary. The measurement of pulse width at half maximum was obtained using a cubic interpolation in order to overcome the limited (albeit high, $100 \mathrm{GS} / \mathrm{s}$ ) sampling rate of the measurement apparatus. For that measurement we used the fastest detection path (Path 2), which enables better resolution of the fastest pulse. However, this prevents the measurements of the smallest values of $\Delta$ for which the intensity variation associated to the excitable orbit is very small (cf. Figure1(A)). Besides the analysis of the response duration, two points are worth mentioning. First, possibly due to the finite risetime of the phase modulator, the efficiency of the perturbation decreases with increased detuning, which reduces the corresponding available sample size. In addition, while a single and well defined response is the hugely dominant behavior, we noticed rare multiple pulses events in response to a single phase perturbation (for detuning values greater than $12 \mathrm{GHz}$ ). Since these multiple pulses consist essentially of repetitions of the single pulses, they are indistinguishable on the bidimensional projection of the reconstructed phase space shown on Figure1. Their relative number increases with the detuning and they become the majority at detuning $17 \mathrm{GHz}$ and decrease afterwards. While the control of these

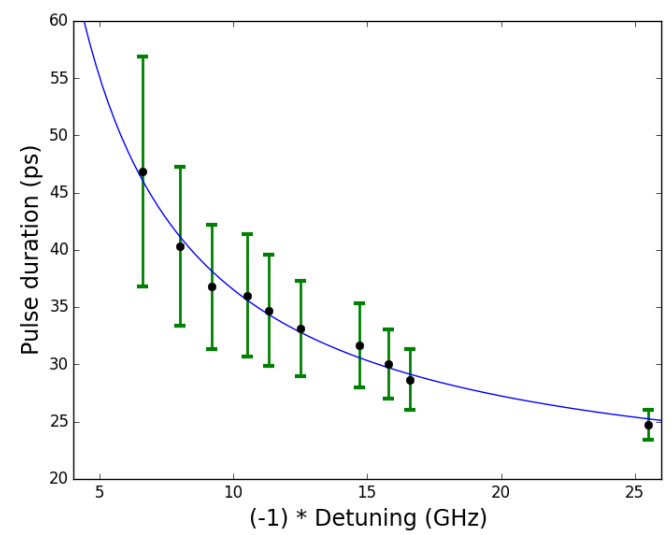

Figure 2. Full width at half maximum of excitable pulses depending on the detuning. Ten to several hundreds of events are considered for the statistics. Black dots: mean of the duration distributions. Green errobars: standard deviation of the distributions. Blue curve: fit of black dots as an inverse function of the detuning $\left(\frac{186}{\Delta}+18\right)$. 


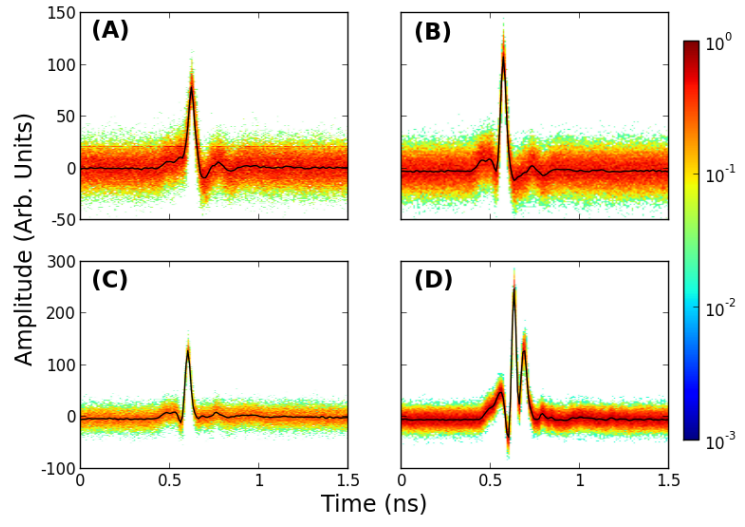

Figure 3. Histograms of the responses observed in Figure 2 in the temporal domain plotted for increasing absolute values of the detuning parameter. Due to different efficiency of the perturbation in these different regimes, the statistical sample size varies between 350 and 600 responses (discussed in the text). Color scale is logarithmic and normalized to the maximum of histograms in the case of unity efficiency. Black curves: Mean of histograms. (A): $\Delta=-6.6 \mathrm{GHz}$ (point 1). (B): $\Delta=-9.2 \mathrm{GHz}$ (point 3). (C): $\Delta=-12.5 \mathrm{GHz}$ (point 6). (D): $\Delta=-25.5 \mathrm{GHz}$ (last point).

multiple pulses (most probably related to homoclinic teeth [Wieczorek et al., 2002]) is of obvious interest, we choose not to consider them in the present work and focus on the duration of a single pulse.

Figure2 shows a clear decrease of pulse duration for increasing detuning, starting from 47 ps for low $\Delta$ down to about 28 ps average. The blue curve, which indicates the fit of an inverse function to the average pulse duration gives a horizontal asymptote for about $18 \mathrm{ps}$, which is the specified response time of the detector. From that, one can infer a minimal pulse duration of about $10 \mathrm{ps}$. Further increase of the detuning (although leading to an apparent additional shortening of the pulse) leads to the formation of a strong ringing following the initial pulse. This ringing was not taken into account for the measurement of the pulse duration, which we deliberately performed only on the first part of the response.

We show on Figure3(A)-(D) histograms of the superposition of temporal excitable responses of Figure2 (respectively $-6.6,-9.2,-12.5$ and $-25.5 \mathrm{GHz}$ ). Panels are plotted in logarithmic color scales, which are normalized to the maximum of histograms in the case of unity efficiency. As for the phase space reconstruction shown in 1, again the use of the logarithmic color scale clearly shows that the response of the system is always very similar between one run and the next. Black lines show the average of the realizations used to build the histograms. Panels (A), (B) and (D) of Figure3 correspond to the case of unity efficiency, with approximately 600 excitable responses. On the other hand, Figure3(C) has been obtained with a lower efficiency
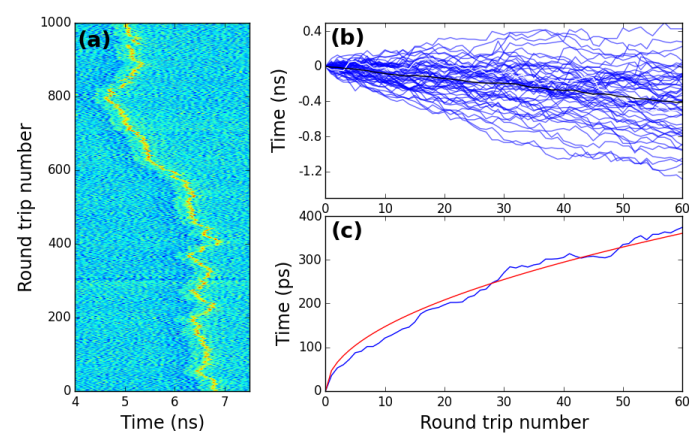

Figure 4. Measure of the temporal diffusion of one topological soliton, acquired for 3500 round trips, 11.32 ns duration each. Parameters are: $I_{s l}=1.376 \mathrm{~mA}, P_{i n j}=1.2 \mu \mathrm{W}, F_{\text {eed }}=2.3 \mu \mathrm{W}$. (a): Slow drift and diffusion of a single topological soliton in the plane ( $\tau_{c}(\mathrm{~ns})$, number of round trips). (b): Superposition of about 60 samples of the difference between the soliton position and the folding as a function of the round trip number, using transparency (0.6); White curve: one realization; Black curve: mean of the trajectories. (c): standard deviation of the distribution defined by samples of panel (b) as a function of the round trip number; Blue curve: measured; Red curve: fit of the blue curve using a square root function.

(about 350 excitable responses).

Figure 3 reveals the unicity of the excitable trajectories in the time domain (as also observed in the phase space reconstruction). One can also notice the modification of the excitable trajectory with parameters, especially the increase of the amplitude. However, Figure3(D) point out a second small ring, visible in the phase space as a closed orbit, immediately following the first one (noticeable only for the last point of Figure2). Then, a modification of the phase space shape with parameters is visible, leading to an intensity dynamics, which is clearly beyond Adler's equation approximation.

\subsection{Localized States: Optical Random Walkers}

In the following we build on the property of neural excitability analyzed above and prepare localized states in a delayed system. We add a space-like dimension to the excitable node prepared previously by inserting a partially reflecting feedback mirror at about two meters from the laser, which reinjects part of the emitted field into the excitable laser.

In presence of this delayed feedback, perturbations applied to the system are not followed by single excitable spikes anymore but result in a periodic emission of spikes. Each spike is extremely similar to the excitable spikes generated in absence of feedback and their repetition period is within $10 \%$ of the roundtrip time of light in the external delay loop. When placed in a comoving reference frame, an observer sees a localized state consisting of a $2 \pi$ phase rotation embedded in a uniformly locked phase state [Garbin et al., 2015]. Similar localized states based on 
an excitable node enclosed in a feedback loop have been observed in a slow-fast resonant tunelling diode [Romeira et al., 2016] and also with a bistable node submitted to delayed feedback, provided the fronts [Giacomelli et al., 2012] are pinned to some external modulation [Marino et al., 2014; Marino et al., 2017]. In the present case, since these localized states consist of optical phase rotations they are analogous to Bloch walls or topological solitons, contrary to the localized states which can be observed in spatially extended systems when switching fronts are pinned to an external ([Haudin et al., 2010] or intrinsic spatial modulation [Barbay et al., 2008]. In a delayed system as the present one, the stability of localized states can be studied by analyzing the Floquet multipliers of a solution and it was found that the number of Floquet multipliers close to unity (ie the number of neutral modes) scales with the number of localized states present in the solution [Garbin et al., 2015]. Each of these neutral modes correspond to the (relative) translation degree of freedom of each localized state. Since all other modes are stable, external perturbations which are not orthogonal to these modes will mostly couple to the translation degree of freedom [Maggipinto et al., 2000]. Therefore, in presence of noise, we expect localized states to wander randomly while still keeping their shape.

We show this phenomenon on Figure4. In this case the optical retroaction applied to the excitable node is delayed by close to $11 \mathrm{~ns}$. A space-time reconstruction as outlined in [Arecchi et al., 1992] is shown on panel a), where we zoom on the position of the localized state within the feedback loop. To further characterize the clearly visible motion of the localized state we split the observed trajectory (over 3500 roundtrips) in 58 samples of 60 roundtrips and plot each of these samples assigning them the same arbitrary origin (panel b). The trajectories show a downward ensemble motion which is due to the chosen reference frame not being strictly comoving with the localized state. This mismatch between the localized state roundtrip frequency and the measurement sampling time only impacts the average position of the system at each discrete time step and is irrelevant for the characterization of the random motion. The trajectories diverge clearly and this divergence can be measured by calculating for each roundtrip the standard deviation of the distribution of positions within the pseudo-space defined by the feedback loop. On panel c) we show that this standard deviation scales likes the square root of the roundtrip number (ie with discrete time), showing that localized states are random walkers which diffuse within the feedback loop. Although a detailed description of how noise projects onto the neutral mode is left out of this work, one intuition of the origin of this motion can be gained by considering that the system is fundamentally an excitable one with delayed retroaction. In an excitable system, the excitable spike itself is essentially insen- sitive to noise due to the strongly attractive nature of the orbit, as discussed above 2.1. However, when an excitable spike is generated, the system undergoes a barrier crossing process and this process is of course very sensitive to noise. Noise has a double impact on this process: broadening the excitability threshold [Pedaci et al., 2010] and adding a random component to the delay in the response of the system to external perturbations [Garbin et al., 2017]. This last part can be expected to cause a random variation in the regeneration time of the excitable spike travelling back and forth in the feedback loop, causing a jitter of the periodic solution.

\section{Conclusion}

An experimental analysis of a laser with an injected signal brought into an excitable regime and submitted to perturbations is reported. A 2-dimensional projection of the phase space is given for different parameters, allowing to distinguish excitable trajectories as large and unique excursions on a cycle going back to the initial stable fixed point. Measuring the response duration, we find that the pulse duration can be taken down to about $10 \mathrm{ps}$ by increasing the detuning. Beyond this value, a way to improve excitable pulses velocity, by increasing the detuning (and the injected power accordingly), is identified. When the excitable system is submitted to delayed retroaction, we observed localized states which form inside the delay loop to undergo a diffusive behavior, which we interpret on the basis of excitability as an essentially noise-insensitive process which follows a very noise-sensitive barrier-crossing process.

\section{Funding Information}

We acknowledge support from Région Provence Alpes Côte d'Azur through grant number DEB 121538 and from Agence Nationale de la Recherche through grant number ANR-12-JS04-0002-01.

\section{References}

Adler, R. (1946). A study of locking phenomena in oscillators. Proceedings of the IRE, 34(6):351-357.

Arecchi, F. T., Giacomelli, G., Lapucci, A., and Meucci, R. (1992). Two-dimensional representation of a delayed dynamical system. Physical Review A, 45(7):-4225.

Barbay, S., Hachair, X., Elsass, T., Sagnes, I., and Kuszelewicz, R. (2008). Homoclinic snaking in a semiconductor-based optical system. Physical Review Letters, 101(25):253902.

Barland, S., Piro, O., Giudici, M., Tredicce, J. R., and Balle, S. (2003). Experimental evidence of van der pol fitzhugh nagumo dynamics in semiconductor optical amplifiers. Phys. Rev. E, 68:036209. 
Coullet, P., Daboussy, D., and Tredicce, J. R. (1998). Optical excitable waves. Phys. Rev. E, 58:5347-5350.

Dubbeldam, J. L. A., Krauskopf, B., and Lenstra, D. (1999). Excitability and coherence resonance in lasers with saturable absorber. Phys. Rev. E, 60(6):6580-6588.

Garbin, B., Dolcemascolo, A., Prati, F., Javaloyes, J., Tissoni, G., and Barland, S. (2017). Refractory period of an excitable semiconductor laser with optical injection. Phys. Rev. E, 95(1):012214.

Garbin, B., Goulding, D., Hegarty, S. P., Huyet, G., Kelleher, B., and Barland, S. (2014). Incoherent optical triggering of excitable pulses in an injectionlocked semiconductor laser. Opt. Lett., 39(5):12541257.

Garbin, B., Javaloyes, J., Tissoni, G., and Barland, S. (2015). Topological solitons as addressable phase bits in a driven laser. Nat Commun, 6.

Garbin, B., Turconi, M., Giudici, M., Tissoni, G., Feyereisen, M., and Barland, S. (2013). Control of excitable pulses in a laser with optical injection. In Laser Dynamics and Nonlinear Photonics, 2013 Sixth "Rio De La Plata” Workshop on, pages 1-3.

Giacomelli, G., Marino, F., Zaks, M. A., and Yanchuk, S. (2012). Coarsening in a bistable system with long-delayed feedback. EPL (Europhysics Letters), 99(5):58005.

Giudici, M., Green, C., Giacomelli, G., Nespolo, U., and Tredicce, J. R. (1997). Andronov bifurcation and excitability in semiconductor lasers with optical feedback. Phys. Rev. E, 55:6414-6418.

Goulding, D., Hegarty, S. P., Rasskazov, O., Melnik, S., Hartnett, M., Greene, G., McInerney, J. G., Rachinskii, D., and Huyet, G. (2007). Excitability in a quantum dot semiconductor laser with optical injection. Phys. Rev. Lett., 98:153903.

Haudin, F., Elías, R. G., Rojas, R. G., Bortolozzo, U., Clerc, M. G., and Residori, S. (2010). Front dynamics and pinning-depinning phenomenon in spatially periodic media. Physical Review E, 81(5):056203.

Hodgkin, A. and Huxley, A. (1952). A quantitative description of membrane current and its application to conduction and excitation in nerve. J. Physiol, 117:500-544

Hodgkin, A. L., Huxley, A. F., and Katz, B. (1952). Measurement of current-voltage relations in the membrane of the giant axon of loligo. J. Physiol., 116:424.

Kelleher, B., Bonatto, C., Huyet, G., and Hegarty, S. P. (2011). Excitability in optically injected semiconductor lasers: Contrasting quantum- well- and quantumdot-based devices. Phys. Rev. E, 83:026207.
Kennel, M. B., Brown, R., and Abarbanel, H. D. (1992). Determining embedding dimension for phase-space reconstruction using a geometrical construction. Physical review A, 45(6):3403.

Kuhnert L., Agladze K. I., and Krinsky V. I. (1989). Image processing using light-sensitive chemical waves. Nature, 337(6204):244-247. 10.1038/337244a0.

Larotonda, M. A., Hnilo, A., Mendez, J. M., and Yacomotti, A. M. (2002). Experimental investigation on excitability in a laser with a saturable absorber. Phys. Rev. A, 65(3):033812.

Maass, W. and Markram, H. (2004). On the computational power of circuits of spiking neurons. Journal of Computer and System Sciences, 69(4):593 - 616.

Maggipinto, T., Brambilla, M., Harkness, G. K., and Firth, W. J. (2000). Cavity solitons in semiconductor microresonators: Existence, stability, and dynamical properties. Phys. Rev. E, 62(6):8726-8739.

Marino, F., Giacomelli, G., and Barland, S. (2014). Front pinning and localized states analogues in long-delayed bistable systems. Phys. Rev. Lett., 112(10):103901.

Marino, F., Giacomelli, G., and Barland, S. (2017). Splitting in the pinning-depinning transition of fronts in long-delayed bistable systems. Phys. Rev. E, 95(5):052204.

Neumann, E. and Bernhardt, J. (1977). Physical chemistry of excitable biomembranes. Ann. Rev. Biochem, 46:117.

Pedaci, F., Huang, Z., van Oene, M., Barland, S., and Dekker, N. H. (2010). Excitable particles in an optical torque wrench. Nat Phys, 7(3):259-264.

Plaza, F., Velarde, M. G., Arecchi, F. T., Boccaletti, S., Ciofini, M., and Meucci, R. (1997). Excitability following an avalanche-collapse process. EPL, 38(2):85.

Romeira, B., Avó, R., Figueiredo, J. M., Barland, S., and Javaloyes, J. (2016). Regenerative memory in time-delayed neuromorphic photonic resonators. Scientific reports, 6.

Takens, F. (1981). Detecting strange attractors in turbulence. In Dynamical systems and turbulence, Warwick 1980, pages 366-381. Springer.

Turconi, M., Garbin, B., Feyereisen, M., Giudici, M., and Barland, S. (2013). Control of excitable pulses in an injection-locked semiconductor laser. Phys. Rev. E, 88:022923.

Wieczorek, S., Krauskopf, B., and Lenstra, D. (2002). Multipulse excitability in a semiconductor laser with optical injection. Phys. Rev. Lett., 88:063901. 